\title{
Cecal Volvulus Following Elective Laparoscopic Cholecystectomy: A Case Report
}

\author{
Yoshiaki Mizuguchi, Yasuhiro Mamada, Tetsuya Shimizu, Tomohiro Kanda, \\ Hiroyasu Furuki, Yuichi Akama, Nobuhiko Taniai, Yoshiharu Nakamura, \\ Masato Yoshioka, Akira Matsushita, Yoichi Kawano and Eiji Uchida
}

Department of Surgery, Nippon Medical School, Tokyo, Japan

Cecal volvulus is characterized by torsion of the cecum around its own mesentery. However, cecal volvulus rarely develops soon after elective laparoscopic cholecystectomy. We report on a case of cecal volvulus that developed in a 54-year-old women 1 day after elective laparoscopic cholecystectomy and was successfully treated via colonoscopic decompression. The symptoms gradually improved in conjunction with recovery from postoperative ileus. Whether the incidence of volvulus has increased with the use of laparoscopic procedures, including laparoscopic cholecystectomy, has yet to be determined. Considering the current trend toward minimally invasive surgery, cecal volvulus should be considered in patients who have postoperative abdominal pain and distention. (J Nippon Med Sch 2016; 83: 172-176)

Key words: cecal volvulus, colonoscopic decompression, laparoscopic cholecystectomy

\section{Introduction}

Since being introduced in the 1980s, laparoscopic cholecystectomy has become the standard treatment for benign diseases of the gallbladder because of its several advantages over open cholecystectomy: less invasiveness, reduced scaring, less-severe postoperative pain, and improved cosmesis. However, laparoscopic cholecystectomy is associated with several possibly life-threatening complications, such as injury to the biliary tract, vessels, and the adjacent bowel ${ }^{1}$. Cecal volvulus is characterized by torsion of the cecum around its own mesentery and often results in obstruction, ischemia, necrosis, and perfora-

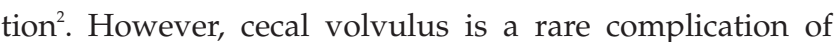
laparoscopic cholecystectomy. Here, we describe a case of cecal volvulus in a 54-year-old woman after laparoscopic cholecystectomy.

\section{Case Presentation}

A 54-year-old woman with a history of mild cholelithiasis and recurrent colicky pain of the right hypochondriac region was referred to our hospital for the treatment of recently worsening symptoms. The patient had undergone open appendectomy for acute appendicitis 40 years earlier and was receiving oral antihypertensive medications. After performing a preoperative physical evaluation and imaging diagnosis via computed tomography (CT) and ultrasonography of the abdomen, we planned elective laparoscopic cholecystectomy with the patient under general anesthesia to remove a $17-\mathrm{mm}$ in length gallstone. The CT revealed mild inflammation of the gallbladder with no wall thickening, swelling, or inflammation of the adjacent tissues. No other abnormalities were found with $\mathrm{CT}$ of the abdomen and pelvis.

For laparoscopic cholecystectomy under general anesthesia, the patient was placed in the supine position with the head slightly elevated and rotated to the left under pneumoperitoneum of $8 \mathrm{~mm} \mathrm{Hg}$ with bicarbonate gas. Three ports were placed: a $12-\mathrm{mm}$ port at the umbilicus to manipulate the 10-mm flexible scope and two $5-\mathrm{mm}$ ports to the right and left of a midclavicular line for use by the operator. A general survey with the laparoscope at the start of the operation found no abnormalities other than mild adhesions between the gallbladder and adjacent structures, and the gallbladder itself was almost completely normal. With an electrode and laparoscopic coagulation shears, the cystic duct and cystic artery were

Correspondence to Yoshiaki Mizuguchi, Department of Surgery, Nippon Medical School, 1-1-5 Sendagi, Bunkyo-ku, Tokyo 1138603, Japan

E-mail: yoshi1224@gmail.com

Journal Website (http://www.nms.ac.jp/jnms/) 
uncovered and clipped. In addition, intraoperative cholangiography was performed but found no abnormalities, such as remnant bile duct stones and bile duct injury. After the gallbladder had been resected, no remnant bleeding or bile leakage was observed, and the wound was closed. No drainage tube was placed. The surgical dura-

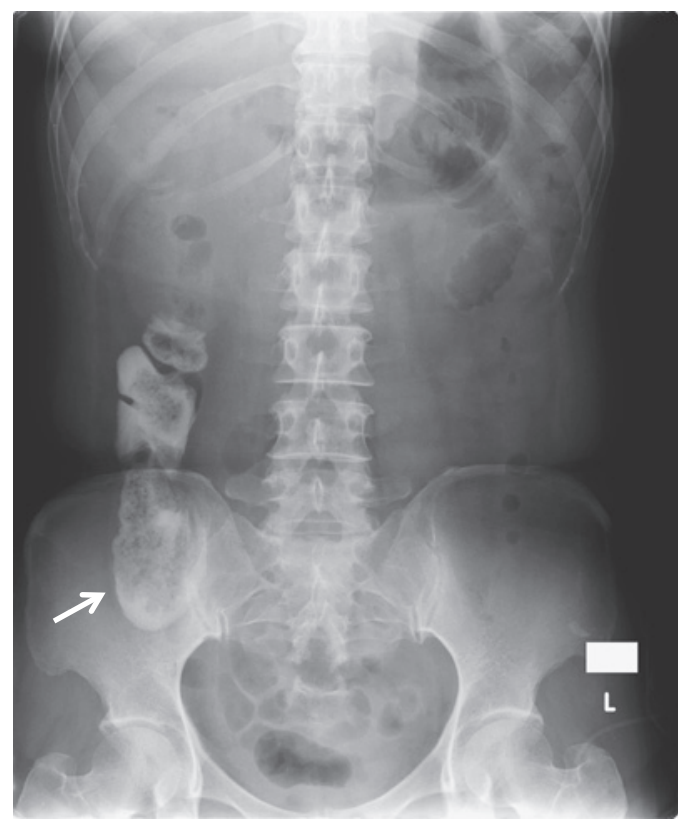

Fig. 1 An X-ray examination on postoperative day 1 showing normal position of the cecum (arrow) and the contrast medium used for intraoperative cholangiography. tion was 76 minutes, and blood loss was minimal.

The patient received intravenous analgesic agents, which included fentanyl. The postoperative course was almost uneventful, and abdominal X-ray images obtained on the morning of the first postoperative day showed no abnormalities (Fig. 1). The contrast medium that had been used for intraoperative cholangiography entered the cecum and ascending colon. However, in the afternoon of the same day the patient complained of exacerbated periumbilical abdominal pain and nausea. Although the patient was afebrile, physical examination revealed a distended abdomen with tenderness in the periumbilical area.

An X-ray examination of the abdomen revealed distention of the cecum in the upper left quadrant, and the contrast medium used for intraoperative cholangiography showed a "bird's beak" deformity of the constricted cecum (Fig. 2A). Furthermore, contrast-enhanced CT confirmed marked distention of the cecum in the left upper quadrant and revealed twisting of the mesenchymal vessel (whirl sign) (Fig. 2B). Neither port site herniation nor bile leakage was indicated. Therefore, acute cecal volvulus was diagnosed, and colonoscopic decompression was emergently performed.

During colonoscopy, moderate inflammation and congestion were noted, but no severe ischemic changes to the colonic mucosa were seen (Fig. 3). In addition, intestinal air and content had flowed from the ileum to the

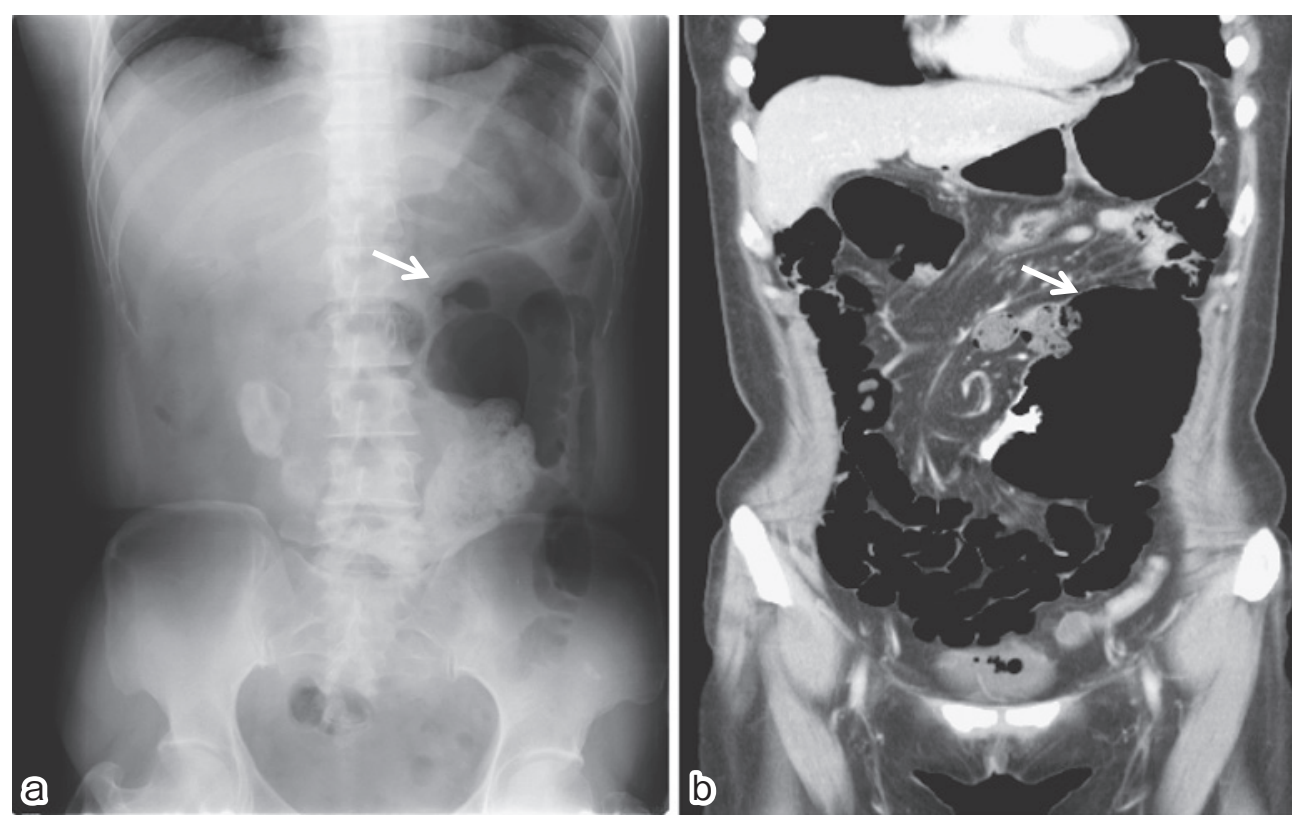

Fig. 2 An X-ray examination and computed tomography examination demonstrating cecal volvulus. An X-ray examination (A) and abdominal computed tomography (B) showing dilatation of the cecum in the left upper quadrant of the abdomen (arrow). 


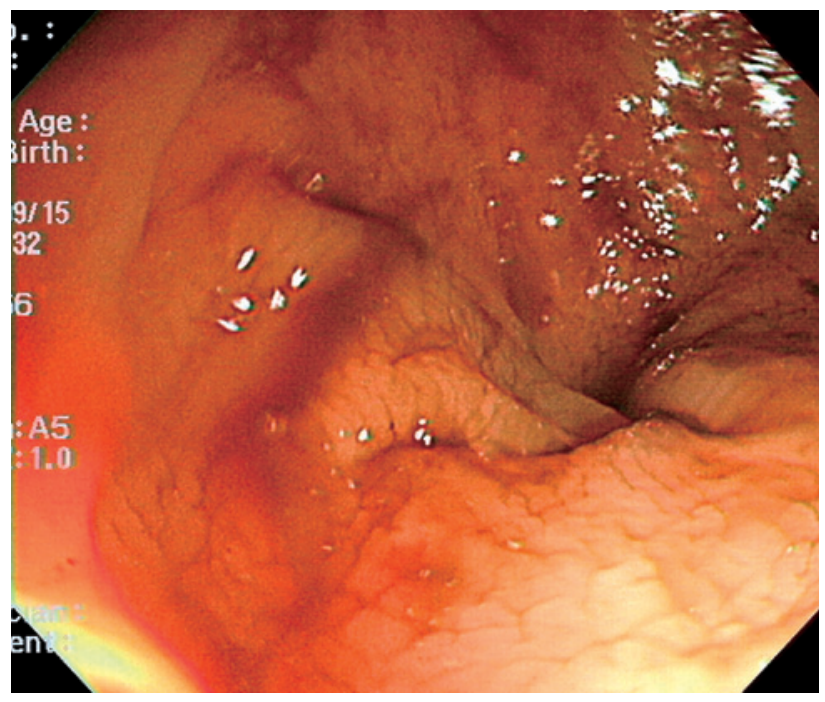

Fig. 3 Photograph of the colonoscopy. Colonoscopy demonstrated dilatation of the cecum without ischemic change to the colonic mucosa. During colonoscopy, air and intestinal content actively flowed into the dilated cecum.

dilated cecum. After colonoscopy, the symptoms had resolved, and a second abdominal CT examination soon after colonoscopic decompression demonstrated that the decompressed cecum and ascending colon were in normal locations (Fig. 4). Although intermittent abdominal pain remained, it gradually improved; the patient was discharged and, at a follow-up examination 48 months after surgery, was found to be well.

\section{Discussion}

We have reported a case of cecal volvulus that occurred soon after elective laparoscopic cholecystectomy. Cecal volvulus is characterized by torsion of the flexible cecum around its own mesentery. Cecal volvulus frequently progresses to obstruction, ischemia, necrosis, perforation, and fecal peritonitis ${ }^{2}$. Cecal volvulus accounts for $15 \%$ to $40 \%$ of cases of colonic volvulus and generally occurs in younger patients (30 to 60 years old) than does sigmoidal volvulus ${ }^{3}$. Immediate surgical reduction of the twisted segment is required to prevent further progression to necrosis and perforation ${ }^{4,5}$. Although the underlying causes of cecal volvulus are often unknown, many etiological and predisposing factors are possible; if a mobile cecum is present, the etiology is most likely multifactorial ${ }^{2}$.

Previous studies have found that mobile cecum is one of the prerequisites for the development of cecal volvulus, in which the failure of peritoneal fixation allows the proximal colon to be free and mobile ${ }^{2,5}$. Another prerequi-

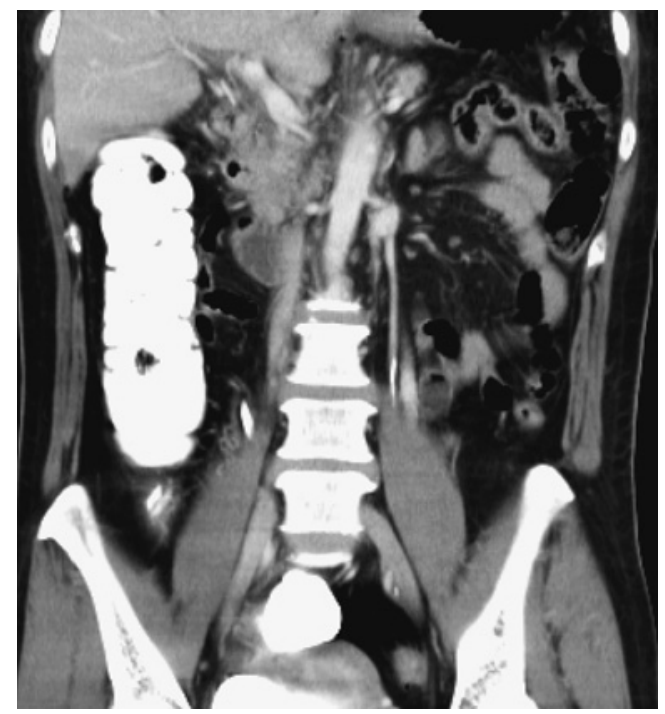

Fig. 4 Computed tomography examination after colonoscopic decompression. After colonoscopy, an computed tomography of the abdomen demonstrated the decompressed cecum in the normal location.

site for cecal volvulus is restriction of the bowel at a fixed point, such as an adhesion, mass, and scarring ${ }^{4}$, within the abdomen which serves as a fulcrum for rotation. A risk factor for cecal volvulus is abdominal surgery, which has preceded $20 \%$ to $50 \%$ of cases ${ }^{5}$. Fibrotic adhesions that develop after surgery can form a point of fixation that allows the cecum to rotate ${ }^{5}$. Appendectomy is known to increase cecal mobility ${ }^{6}$. Dissection during appendectomy can free peritoneal attachments, mobilize parts of the ascending colon and terminal ileum, and sufficiently increase cecal mobility to form a volvulus. Because the appendectomy the present patient underwent was open, it might have increased the risk of adhesion formation and created a fixed point within the abdomen; however, routine inspection of the abdominal cavity at the end of the surgery revealed no abnormalities or adhesions. Nonetheless, because cecal volvulus is rarely a postoperative complication, no guidelines have been widely accepted for the systemic assessment of the abdominal cavity, especially the omentum, colon, and cecum, at the end of laparoscopic surgery.

Other factors that have been suggested to increase the risk of cecal volvulus include chronic constipation, postoperative ileus, distal colonic obstruction, late-term pregnancy, and high fiber intake $e^{4,5}$. Of these factors, proximal colon dilatation caused by postoperative ileus related to surgical stress may have played an important role in our patient. Peristalsis of the small intestine has been re- 
Table 1 Reported cases of cecal volvulus after laparoscopic surgery

\begin{tabular}{|c|c|c|c|c|c|c|c|}
\hline Case report & $\begin{array}{l}\text { Patient } \\
\text { age }\end{array}$ & Sex & Operation & $\begin{array}{l}\text { Time } \\
\text { after } \\
\text { surgery }\end{array}$ & Postoperative treatment & Imaging & $\begin{array}{l}\text { Predisposing } \\
\text { factor }\end{array}$ \\
\hline Duron et al ${ }^{11}$ & - & - & Cholecystectomy & $72 \mathrm{~h}$ & Surgery & - & $\begin{array}{l}\text { Unrecognized } \\
\text { malrotation }\end{array}$ \\
\hline Bariol et al ${ }^{12}$ & $74 \mathrm{y}$ & $\mathrm{F}$ & Cholecystectomy & $36 \mathrm{~h}$ & Right hemicolectomy & - & $\begin{array}{l}\text { History of } \\
\text { appendectomy } \\
\text { and hysterectomy }\end{array}$ \\
\hline Morris et al ${ }^{13}$ & $71 \mathrm{y}$ & $\mathrm{F}$ & Cholecystectomy & $15 y$ & Ileocecectomy & $\begin{array}{l}\text { X-ray, } \\
\text { CT }\end{array}$ & $\begin{array}{l}\text { Adhesion forma- } \\
\text { tion with spilled } \\
\text { gallstones }\end{array}$ \\
\hline Present case & $43 y$ & $\mathrm{~F}$ & Cholecystectomy & $24 \mathrm{~h}$ & Colonoscopy & $\begin{array}{l}\text { X-ray, } \\
\text { CT }\end{array}$ & $\begin{array}{l}\text { History of } \\
\text { appendectomy }\end{array}$ \\
\hline Agahi and Harle ${ }^{14}$ & $56 y$ & $\mathrm{~F}$ & $\begin{array}{l}\text { Adjustable } \\
\text { gastric banding }\end{array}$ & $2 y$ & $\begin{array}{l}\text { Surgery: excision and } \\
\text { anastomosis }\end{array}$ & $\begin{array}{c}\text { X-ray, } \\
\text { CT }\end{array}$ & $\begin{array}{l}\text { Adjustable gastric } \\
\text { band }\end{array}$ \\
\hline Mclntosh et al ${ }^{15}$ & $33 \mathrm{y}$ & $\mathrm{F}$ & Appendectomy & $48 \mathrm{~h}$ & Cecopexy & X-ray & $\begin{array}{c}\text { Present } \\
\text { appendectomy }\end{array}$ \\
\hline Eng and Ravindra ${ }^{10}$ & $63 \mathrm{y}$ & $\mathrm{F}$ & $\begin{array}{c}\text { Kidney } \\
\text { transplantation }\end{array}$ & $4 \mathrm{w}$ & $\begin{array}{l}\text { Laparotomy, right } \\
\text { hemicolectomy }\end{array}$ & $\begin{array}{l}\text { X-ray, } \\
\text { CT }\end{array}$ & $\begin{array}{l}\text { History of } \\
\text { nephrectomy }\end{array}$ \\
\hline Kim et $\mathrm{al}^{6}$ & $73 \mathrm{y}$ & M & $\begin{array}{l}\text { Ventral hernia } \\
\text { repair }\end{array}$ & $96 \mathrm{~h}$ & $\begin{array}{l}\text { 1) Surgical resection and } \\
\text { anastomosis, 2) ileostomy }\end{array}$ & $\begin{array}{c}\text { X-ray, } \\
\text { CT }\end{array}$ & $\begin{array}{l}\text { History of } \\
\text { appendectomy }\end{array}$ \\
\hline
\end{tabular}

ported to recover earlier after surgery (12-24 hours) than does peristalsis of the colon (48-120 hours $)^{7}$. Cecal volvulus likely develops about 24 hours after surgery, as demonstrated in our patient by the normal location of the cecum on abdominal X-ray images obtained in the morning of postoperative day 1 and by the start of abdominal pain that afternoon. Consequently, the cecal volvulus likely developed and progressed because the peristalsis of the colon recovered later than that of the intestine. In fact, during emergency colonoscopy in our patient, we observed that air and intestinal content had flowed into the dilated cecum. We suspect that the excessive stress to the paralytic cecum caused by the air and content flowing from the small intestine, whih recovered earlier, resulted in cecal dilatation and the subsequent cecal volvulus. A previous study has noted that air insufflation during colonoscopy leads to cecal dilatation and might play a role in the development of cecal volvulus ${ }^{8,9}$. These observations suggest that even colonoscopy with insufflation of air can lead to cecal dilatation and subsequent volvulus of a mobile cecum, as might have occurred in the present case.

A search of the PubMed database (http://www.ncbi. nlm.nih.gov/pubmed) confirmed that cecal volvulus after laparoscopic cholecystectomy is rare and that only 3 cases have previously been reported in the Englishlanguage literature (Table 1). In these cases and the present case, the patients had a mean age of 63 years (range, 43-74 years) and 3 of the 4 patients were women (the sex of 1 earlier patient is unknown). Three of these patients had cecal volvulus 24 to 36 hours after surgery, and 1 patient had inflammation caused by spilled gallstones 15 years after surgery. All previous patients underwent surgical treatment for cecal volvulus. Two of the 4 patients had previously undergone open appendectomy. In addition, few cases of cecal volvulus have been reported to occur after laparoscopic procedures for appendectomy, gastric banding, nephrectomy, or laparoscopic ventral hernia repair (Table 1). Because of the recent observation that cecal volvulus has become more common as laparoscopic surgery has more commonly been performed, some authors have hypothesized that laparoscopic surgery is associated with an increased risk of cecal volvulus ${ }^{8}$. Laparoscopy, pneumoperitoneum, patient positioning, lateral tilting of the operating table, and mobilization of parts of the right colon have been suggested to be causative factors for cecal volvulus in patients with a mobile cecum ${ }^{10}$. To detect cecal volvulus CT is highly recommended because of its high diagnostic value, but plain abdominal radiography is not recommended because visual signs of cecal volvulus, such as a beak sign, are often unrevealed ${ }^{3}$.

In the present case, cecal volvulus was suggested by both CT and X-ray examinations. Interestingly, the contrast medium used for intraoperative cholangiography demonstrated the classic beak sign. Colonoscopic decompression followed by conservative therapy was successful, and the symptoms spontaneously improved, prob- 
ably as colonic decreased. After the cecum decompressed with colonoscopy on postoperative day 2 , the symptoms remained, but the location of the cecum on X-ray images continued to improve.

Because colonoscopic decompression is less invasive than surgery for treating cecal volvulus, we believe that if a patient's general condition is stable and emergency surgery is not indicated, colonoscopy might be more beneficial. However, remember that recurrence rates after colonoscopic decompression are higher in cases with cecal volvulus and that surgical treatment is required to prevent gangrene and perforation ${ }^{8}$. Therefore, in patients who have postoperative abdominal pain and distention early after laparoscopic surgery, cecal volvulus should be considered to avoid delayed diagnoses and negative outcomes, including death.

Conflict of Interest: The authors declare that there is no conflict of interest related to this article.

\section{References}

1. Deziel DJ, Millikan KW, Economou SG, Doolas A, Ko ST, Airan MC: Complications of laparoscopic cholecystectomy: a national survey of 4,292 hospitals and an analysis of 77,604 cases. Am J Surg 1993; 165: 9-14.

2. Hasbahceci M, Basak F, Alimoglu O: Cecal volvulus. Indian J Surg 2012; 74: 476-479.

3. Moore CJ, Corl FM, Fishman EK: CT of cecal volvulus: unraveling the image. AJR Am J Roentgenol 2001; 177: 95-98.

4. O'Mara CS, Wilson TH Jr, Stonesifer GL, Cameron JL: Cecal volvulus: analysis of 50 patients with long-term follow-up. Ann Surg 1979; 189: 724-731.

5. Consorti ET, Liu TH: Diagnosis and treatment of caecal volvulus. Postgrad Med J 2005; 81: 772-776.

6. Kim J, Nguyen S, Leung P, Divino C: Cecal bascule after laparoscopic ventral hernia repair. JSLS 2013; 17: 484-486.

7. Rothnie NG, Harper RA, Catchpole BN: Early postoperative gastrointestinal activity. Lancet 1963; 2: 64-67.

8. Halabi WJ, Jafari MD, Kang CY, Nguyen VQ, Carmichael JC, Mills S, Pigazzi A, Stamos MJ: Colonic volvulus in the United States: trends, outcomes, and predictors of mortality. Ann Surg 2014; 259: 293-301.

9. Agko M, Gociman B, Keilani ZM, Mukherjee A: Cecal volvulus: a rare complication of colonoscopy. Int J Colorectal Dis 2012; 27: 265-266.

10. Eng $\mathrm{M}$, Ravindra K: Cecal volvulus following laparoscopic nephrectomy and renal transplantation. JSLS 2009; 13: 612-615.

11. Duron JJ, Hay JM, Msika S, Gaschard D, Domergue J, Gainant A, Fingerhut A: Prevalence and mechanisms of small intestinal obstruction following laparoscopic abdominal surgery: a retrospective multicenter study. French Association for Surgical Research. Arch Surg 2000; 135: 208-212.

12. Bariol SV, McEwen HJ: Caecal volvulus after laparoscopic cholecystectomy. Aust N Z J Surg 1999; 69: 79-80.

13. Morris MW Jr, Barker AK, Harrison JM, Anderson AJ, Vanderlan WB: Cicatrical cecal volvulus following laparoscopic cholecystectomy. JSLS 2013; 17: 333-337.

14. Agahi A, Harle R: A serious but rare complication of laparoscopic adjustable gastric banding: bowel obstruction due to caecal volvulus. Obes Surg 2009; 19: 1197-1200.

15. McIntosh SA, Ravichandran D, Wilmink AB, Baker A, Purushotham AD: Cecal volvulus occurring after laparoscopic appendectomy. JSLS 2001; 5: 317-318.

(Received, November 14, 2015)

(Accepted, February 12, 2016) 